\title{
Les effets d'un programme de conscience morphologique sur l'identification des mots en français
}

\author{
Anila Fejzo, Lucie Godard, Line Laplante \\ Université du Québec à Montréal, Canada
}

Article reçu le 2 mai 2014, a accepté 16 Septembre 2014, version final 27 Octobre 2014

\begin{abstract}
Un nombre de plus en plus important d'études ont démontré que l'entrainement de la conscience morphologique contribue au développement de la littératie, notamment de l'identification des mots. Or, cette contribution a été vérifiée seulement auprès d'élèves en langue première (L1). Comme une telle contribution dépend de la manière dont une langue encode la morphologie, dans un contexte de langue seconde (L2), particulièrement quand celle-ci encode la morphologie différemment de la L1, cette contribution doit être vérifiée. Notre étude vise à examiner les effets de l'entrainement de la conscience morphologique sur l'identification des mots plurimorphémiques chez des élèves arabophones de $3 \mathrm{e}$ et de $4 \mathrm{e}$ année scolarisés en français. Cinquante-deux élèves ont participé à cette étude quasi-expérimentale. Pendant dix semaines, les élèves du groupe expérimental ont suivi un programme de conscience morphologique qui portait sur les familles de mots, le sens et les règles de jonction des affixes les plus productifs en français. Des mesures de la conscience morphologique et de l'identification des mots ont été prises auprès des deux groupes au prétest et au post-test. Nous avons également mesuré la conscience phonologique, la denomination rapide et les capacités cognitives des participants afin de mieux isoler les effets de l'intervention. Les analyses statistiques ont montré que les élèves du groupe experimental ont performé significativement mieux que ceux du groupe contrôle dans la précision de l'identification des mots plurimorphémiques. Ces résultats corroborent les résultats de recherches en L1 et suggèrent que l'entrainement de la conscience morphologique pourrait être une piste prometteuse pour soutenir les lecteurs en L2.
\end{abstract}

\section{Introduction}

Depuis les deux dernières décennies, plusieurs chercheurs se sont penchés sur la relation entre la conscience morphologique et l'apprentissage de la lecture, notamment l'identification des mots écrits. Cette conscience qui désigne la capacité à analyser la structure morphologique des mots et l'habileté à la manipuler (Carlisle, 1995) semble avoir une relation étroite avec l'identification des mots. Les évidences scientifiques d'une telle relation sont d'autant plus convaincantes qu'elles proviennent d'études inscrites dans une pluralité de perspectives et de devis de recherche. 
Ainsi, dans une perspective fonctionnelle, certaines études (Severine Casalis, Dusautoir, Cole, \& Ducrot, 2009; Deacon, Parrila, \& Kirby, 2006; Nagy, Berninger, \& Abbot, 2006) ont vérifié si l'identification des mots plurimorphémiques est influencée, en termes de temps et de précision, par une amorce morphologique. Les résultats de ces études mettent en évidence le fait que les lecteurs lisent plus rapidement et avec plus de précision les mots qui leur sont présentés lorsque ceux-ci sont précédés d'une amorce morphologique que lorsqu'ils sont précédés d'amorces phonologique ou orthographique (Casalis et al. 2009). Ces résultats, qui suggèrent un effet facilitateur de l'accès aux morphèmes dans l'identification des mots écrits, appuient l'hypothèse d'une interaction entre la conscience morphologique et les processus d'identification des mots au cours de l'apprentissage de la lecture (Seymour, 2005).

Dans une autre perspective, plusieurs études explorent la corrélation entre la conscience morphologique et l'identification des mots à différentes étapes de l'apprentissage de la lecture (Carlisle, 1995, 2000; Séverine Casalis \& Louis-Alexandre, 2000; Colé, Royer, Leuwers, \& Casalis, 2004; Deacon \& Kirby, 2004; Fowler \& Liberman, 1995; Kirby et al., 2011; Schwiebert, Green, \& McCutchen, 2002; Verhoeven $\&$ Schreuder, 2011). Ces études, menées auprès de différentes populations d'élèves, dans lesquelles on retrouve des devis de recherche longitudinaux ou transversaux, apportent un éclairage quant à la trajectoire développementale de cette relation. Nous en dégageons deux hypothèses qui intéressent particulièrement cette recherche. La première hypothèse est à l'effet que la contribution de la conscience morphologique dans l'identification des mots augmenterait avec le niveau scolaire, c'est-à-dire qu'elle jouerait un plus grand rôle aux niveaux scolaires intermédiaires et supérieurs qu'au début de la scolarité (Kirby et al., 2011; Mahony, Singson, \& Mann, 2000; Singson, Mahony, \& Mann, 2000). Plus précisément, il ressort de ces études que la $3^{\mathrm{e}}$ et la $4^{\mathrm{e}}$ année constitueraient une période charnière pour la mise en place de cette contribution. La seconde hypothèse suppose que la contribution de la conscience morphologique influencerait d'abord la précision et, ultérieurement, la rapidité de l'identification des mots morphologiquement complexes (Nagy et al., 2006). Toutefois, ces études corrélationnelles ne peuvent pas nous renseigner sur la nature ni la direction de cette relation. 
Enfin, dans une perspective causale, certaines études proposent des programmes d'entrainement de la conscience morphologique et mesurent leurs effets sur l'identification des mots écrits (Elbro \& Arnbak, 1996; Lyster, 2002; Nunes, Bryant, \& Olsson, 2003). Ces études démontrent que l'entraînement de la conscience morphologique contribue positivement à l'identification des mots. De telles études étant peu nombreuses, la relation de causalité entre la conscience morphologique et l'identification des mots doit être davantage documentée (Carlisle, 2010) à plusieurs égards. Tout d'abord, la méta-analyse réalisée par Carlisle (2010) met en évidence la nécessité de renforcer la validité interne et externe de ces études par le recours rigoureux à des tests adaptés au contenu des programmes d'intervention, susceptibles de mesurer avec précision leurs effets. Ensuite, une description plus détaillée des interventions mises en œuvre permettrait de mieux informer les milieux de la recherche et de l'éducation quant aux modalités ayant conduit aux résultats obtenus. Par ailleurs, la relation causale entre la conscience morphologique et l'identification des mots a été vérifiée seulement auprès d'élèves scolarisés dans leur langue maternelle, en l'occurrence en anglais et en norvégien. Aucune étude, à notre connaissance, n'a mesuré les effets d'un entrainement visant la conscience morphologique auprès d'une population scolarisée dans une langue autre que sa langue maternelle. Questionner les effets de l'entrainement de la conscience morphologique sur l'identification des mots en français et dans un contexte de langue seconde découle, d'une part, de l'hypothèse que l'interaction entre la conscience morphologique et l'identification des mots est tributaire des caractéristiques de la langue ou des langues à l'étude (Seymour, 2005). Nous en traiterons en détail dans la section suivante. D'autre part, ce questionnement revêt une importance particulière dans le contexte où les lecteurs scolarisés en langue seconde semblent avoir plus de difficulté que leurs pairs en L1 (Braunger \& Lewis, 1998) et plus particulièrement les arabophones qui apprennent l'anglais (Randall \& Meara, 1988) et le français (Besse, Demont, \& Gombert, 2007). C'est dans cette optique scientifique que s'inscrit notre étude qui a pour objectif de vérifier les effets d'un programme de conscience morphologique sur l'identification des mots écrits chez des élèves arabophones de $3^{\mathrm{e}}$ et de $4^{\mathrm{e}}$ année du primaire scolarisés en français. 


\section{Contribution de la conscience morphologique dans l'identification des mots écrits en français}

Si le processus qui conduit à l'utilisation des connaissances morphologiques lors de l'identification des mots morphologiquement complexes est loin d'être clair pour les chercheurs (Sénéchal \& Kearnan, 2007; Verhoeven \& Schreuder, 2011), la recherche apporte des preuves convaincantes à l'effet que la conscience morphologique joue un rôle significatif dans la capacité à identifier ces mots (Verhoeven \& Perfetti, 2003). Or, l'identification des mots étant une capacité mesurée en termes de rapidité et de précision, comment expliquer la contribution de la conscience morphologique pour chacun de ces deux aspects?

Pour ce qui est de la rapidité, cette contribution pourrait être expliquée dans le cadre de l'hypothèse des deux voies d'accès lexical (Chialant \& Caramazza, 1995). Selon cette hypothèse, il existerait la voie d'accès direct à la forme globale du mot, comme c'est le cas des mots morphologiquement complexes connus par le lecteur, et la voie de décomposition qui consiste à segmenter les mots en constituants morphémiques et qui est mise en œuvre lors de l'identification des mots morphologiquement complexes non familiers au lecteur. Pour les mots plurimorphémiques à structure orthographique transparente, les deux voies sont activées proportionnellement à leur fréquence, c'est-àdire pour identifier un mot fréquent, la voie d'accès direct à la forme globale est privilégiée, en revanche, pour un mot rarement rencontré, la voie d'accès aux morphèmes décomposés se met en place. En effet, les résultats de Deacon, Whalen et Kirby (2011) ont montré que les élèves de $4^{\mathrm{e}}$, de $6^{\mathrm{e}}$ et de $8^{\mathrm{e}}$ années ont lu plus rapidement et avec plus de précision les mots plurimorphémiques de basse fréquence formés d'une base de haute fréquence que ceux qui dérivaient d'une base de basse fréquence. Ces résultats suggèrent le recours à la voie de décomposition en unités morphémiques. Par contre, la fréquence de la base n'a pas eu d'effet sur la rapidité de l'identification des mots de haute fréquence, ce qui appuie l'hypothèse de l'accès direct à la forme globale du mot pour ce type d'item. De même, Casalis et al. (2009), ayant observé que la rapidité de l'identification des mots plurimorphémiques est significativement influencée par les stimuli morphologiques, ont conclu qu'un niveau de traitement morphologique est mis en 
place lors de l'identification des mots plurimorphémiques chez des lecteurs français de $4^{\mathrm{e}}$ année du primaire.

La contribution de la conscience morphologique dans la rapidité de l'identification des mots plurimorphémiques s'expliquerait par le fait que le lecteur recourt à un nombre réduit de morphèmes composant le mot au lieu de décoder, de façon séquentielle, un plus grand nombre de graphèmes, et ce, peu importe le système d'écriture alphabétique (ex., dans le mot prédire, il y a deux morphèmes et six phonèmes). Or, la contribution de la conscience morphologique dans la précision de l'identification des mots semble être sujette aux contraintes imposées par la manière dont la morphologie est encodée dans une langue donnée (Verhoeven \& Perfetti, 2011). Dans le cas du français, cet encodage est tributaire de l'interaction de deux principes fondamentaux, à savoir phonographique et sémiographique, qui régissent son orthographe (Jaffré \& Fayol, 1997, 2006). Le principe phonographique, qui renvoie à l'encodage des sons à l'écrit, se reflète dans les correspondances entre130 graphèmes et 36 phonèmes (Catach, 1978). Cette discordance numérique engendre le phénomène d'inconsistance des correspondances graphèmes-phonèmes (CGP) (Ziegler \& Goswami, 2005) qui s'avère double en français: le même graphème correspond à différents phonèmes (par exemple le graphème $\mathrm{c}$ correspond à $/ \mathrm{s} / \mathrm{ou} / \mathrm{k} /$ ) et le même phonème est encodé par plusieurs graphèmes (ex., le son /s/ est réalisé par $s, s c, t$, etc). Ce phénomène qui caractérise presque la moitié des graphèmes en français (selon Catach (1978), seulement 70-75 des graphèmes sont stables) exige du lecteur la mise à contribution de diverses connaissances qui concernent l'identification des mots en français. Pour une bonne partie de ces mots, il s'agit de connaissances relevant du deuxième principe, soit le principe qui régit l'encodage du sens à l'écrit. Ce type d'encodage est fortement contrôlé par le principe morphographique (Jaffré, 1999), lequel permet l'identification précise des mots quand l'application des règles de CGP ne suffit pas. Par exemple, content ne sera pas lu de la même façon dans les groupes suivants : ils content et $i$ l est content. L'analyse morphologique de ces mots (le graphème - ent correspondant au suffixe verbal du pluriel dans le premier exemple est muet, alors que ce graphème se prononce /ã/ dans le second) est nécessaire pour leur identification précise. Le lecteur du français est donc incité par le 
double encodage à l'écrit à analyser les constituants morphémiques des mots pour leur attribuer les formes phonologiques respectives.

De plus, la contribution de la conscience morphologique dans la précision de l'identification des mots plurimorphémiques peut être expliquée dans le cadre du modèle de lecture de l'expérience avec le mot (word-experience model of reading) (Reichle \& Perfetti, 2003) qui postule que l'expérience avec les mots aide le lecteur à construire des représentations de haute qualité qui comprennent l'encodage des informations orthographiques, phonologiques et sémantiques. Ces chercheurs précisent que chez les bons lecteurs, des connaissances morphologiques sont également encodées pour représenter les mots plurimorphémiques. Les représentations ainsi construites permettent à ceux-ci d'identifier avec précision les mots plurimorphémiques. D'ailleurs, les résultats de Casalis et al. (2009) obtenus auprès d'élèves francophones qui lisent avec plus de précision les mots quand ils sont précédés par une amorce morphologique suggèrent que les lecteurs du français mettent à profit leurs connaissances morphologiques pour lire avec précision les mots écrits. Ce cadre théorique justifie les recommandations de chercheurs français (Gombert et al. 2002) à l'effet qu'il faut enseigner la conscience morphologique pour soutenir le lecteur du français.

\section{Contribution de la conscience morphologique dans l'identification des mots écrits chez les arabophones}

L'argumentation qui précède porte sur la contribution de la conscience morphologique dans l'identification des mots en français dans le contexte d'une langue première (L1). Or, la présente étude, menée auprès d'une population arabophone scolarisée en français, doit tenir compte du fait que l'identification des mots dans une langue autre que la langue maternelle, que nous appelons ici langue seconde (L2), est influencée par la distance linguistique entre les systèmes graphiques de la L1 et de la L2. Selon Geva (2009) et Koda (2004), plus les langues sont similaires, plus il est facile pour l'apprenant de transférer les habiletés acquises en L1 dans l'identification des mots en L2. Plus les deux langues sont distantes, plus ce transfert sera complexe et difficile pour le lecteur en L2.

À cet égard, il s'avère intéressant d'examiner les résultats de l'étude de Besse et al. (2007). L'étude a été menée auprès de 153 élèves tunisiens de $3^{\mathrm{e}}, 4^{\mathrm{e}}$ et $5^{\mathrm{e}}$ années, et de 
125 élèves brésiliens de $3^{\mathrm{e}}, 5^{\mathrm{e}}$ et $7^{\mathrm{e}}$ années dans une perspective évolutive et comparative. Les deux groupes apprenaient le FL2. Des tests mesurant la capacité d'identification des mots écrits, la compréhension de phrases écrites, les connaissances phonologiques, morphologiques et syntaxiques en L1 et L2 ont été administrés en arabe, en portugais et en français. Les résultats indiquent qu'en identification des mots en français, les arabophones éprouvent plus de difficultés que leurs pairs lusophones, même après le contrôle de leurs connaissances lexicales. Ces résultats plaident en faveur de l'hypothèse à l'effet que l'identification des mots en L2 est influencée par la distance linguistique entre la L1 et la L2.

Il en découle que le rôle de la conscience morphologique dans le développement de l'identification des mots chez la population de notre étude résulterait de l'interaction de facteurs reliés au développement de ces processus dans les deux langues et à la distance linguistique entre le français (L2) et l'arabe (L1). Pour comprendre ce rôle dans l'identification des mots dans une première langue, nous nous référons à certains modèles théoriques (Ehri, 1999, 2005; Seymour, 1997, 2005) qui postulent que pour identifier les mots, le lecteur recourt à des représentations orthographiques, phonologiques et sémantiques stockées en mémoire. Les représentations orthographiques résultent de l'encodage de séquences de lettres de plus en plus longues rencontrées fréquemment lors de la lecture (Ehri, 2005). La construction de ces représentations permet au lecteur de traiter ces séquences comme un tout au lieu de les assembler en utilisant les correspondances graphèmes-phonèmes. Lorsque les séquences de lettres reconnues instantanément correspondent à des morphèmes (ex. avant-cour-eur), le lecteur est entré dans la dernière phase du développement des processus d'identification des mots, qui commence à la fin de la deuxième année (Ehri, 1999). La construction des représentations orthographiques des morphèmes se fait en interaction avec les capacités de segmentation de la structure des mots au niveau morphémique et les connaissances relatives aux règles de jonction des morphèmes propres à une langue donnée (Seymour, 1999). Dans le cas de figure d'un lecteur qui lit dans deux langues, la construction des représentations orthographiques devrait être facilitée ou entravée selon que les langues se ressemblent ou se distinguent de la manière dont elles encodent les morphèmes à l'écrit 
(Seymour, 2005). Pour comprendre la situation des participants de cette étude, nous examinons la manière d'encoder les morphèmes en français et en arabe. Si le français encode les morphèmes de façon linéaire préfixe(s)-racine-suffixe(s) (dé-coll-age-s), cette linéarité est impossible dans l'arabe écrit. En effet, la racine des mots arabes est une structure morphologique composée de trois ou quatre consonnes et les affixes correspondent à des schèmes vocaliques qui s'enchevêtrent avec la racine et donnent majoritairement des structures canoniques de deux syllabes (McCarthy \& Prince, 1990). Par exemple, la base $k-t-b$, dont le sens général est écrire, combinée avec le schème du lieu - $i$-aa-produirait KiTaaB - livre. Compte tenu de la divergence reliée à l'encodage des morphèmes, nous présumons que la construction des représentations orthographiques des mots plurimorphémiques en français s'avère plus complexe pour les élèves arabophones et par conséquent, ils devraient avoir plus de difficulté à les identifier que leurs pairs en FL1 ou d'autres populations en L2 dont la distance entre leur L1 et L2 est moins grande. En effet, les résultats de Fejzo, Godard et Laplante (2013, soumis) montrant que les participants arabophones scolarisés en français, comparés à leurs pairs francophones, réussissent aussi bien les mots courts mais moins bien les mots plurimorphémiques, vont dans ce sens. Ces résultats soutiennent les recommandations de certains chercheurs (Randall et Meara, 1988; Besse et al., 2007) à l'effet qu'il est nécessaire d'intervenir en conscience morphologique pour faciliter l'identification des mots écrits chez des élèves arabophones scolarisés en L2. Si cette nécessité découle des particularités d'encodage des morphèmes en français et des besoins de la population arabophone en L2, les effets d'une telle intervention restent à vérifier. La présente étude vise donc à évaluer les effets d'une intervention en conscience morphologique sur l'identification des mots plurimorphémiques, en termes de précision et de rapidité, chez des élèves arabophones de $3^{\mathrm{e}}$ et de $4^{\mathrm{e}}$ année du primaire scolarisés en français. Au regard de cet objectif, nous nous attendons à ce que le groupe ayant bénéficié du programme d'intervention ait une performance significativement supérieure à celle du groupe contrôle en identification des mots plurimorphémiques. 


\section{Intervention en conscience morphologique}

Afin de préciser les modalités de l'intervention en conscience morphologique à proposer dans cette étude, nous nous sommes basées sur des études similaires à la nôtre à certains égards, sur des écrits scientifiques reliés à la conscience morphologique et à l'enseignement explicite en général, et sur des méta-analyses. Ainsi, menée en contexte francophone, l'étude de Casalis (2007) éclaire quant à la manière de travailler les morphèmes : cibler les morphèmes les plus productifs et les travailler en profondeur. Pour ce qui est de la fréquence et de la durée des activités, Nunes et al. (2003) ont réalisé douze activités de 30 minutes une fois par semaine avec des élèves de $3^{\mathrm{e}}$ et de $4^{\mathrm{e}}$ année. D'autres recherches causales (Elbro et Arnbak, 1996) et des écrits scientifiques (Bowers, 2009; Gombert et al., 2000) ont été analysés pour dégager le type d'activités morphologiques à inclure dans le programme: la dérivation, l'extraction de la base, l'analyse morphologique et le transfert des connaissances morphologiques dans l'identification des mots écrits. Par ailleurs, l'ordre de présentation des activités du programme porte l'empreinte de l'opérationnalisation du concept conscience morphologique de Fejzo (2011). Les activités du programme sont donc proposées dans l'ordre qui suit : 1) les familles de mots, 2) la structure morphologique des mots, 3) les préfixes dé-, in-, a-, re-, 4) la consolidation des connaissances sur les préfixes, 5) des règles de jonction des préfixes, 6) les suffixes nominaux -tion, -age, -eur, -issement, ette, -ée, 7) la consolidation des connaissances sur les suffixes nominaux, 8) les suffixes adjectivaux, -eux, -ique, -ant, 9) les morphogrammes, 10) la dérivation de pseudo-mots. De plus, deux activités d'analyse morphémique sont réalisées en devoir à la maison. L'élagage de l'aspect flexionnel provient du fait que les élèves développent implicitement des connaissances morphologiques flexionnelles dès le début de leur scolarité (Carlisle, 1995; Gombert et al. 2000), mêmes les élèves arabophones scolarisés en français (Fejzo, 2005). Outre les activités explicitement morphologiques, en modalité orale ou écrite, le programme contient un deuxième volet, quoique modeste, qui invite les élèves à exploiter les connaissances et les habiletés morphologiques lors de la lecture des mots plurimorphémiques, comme il est fortement suggéré dans la littérature à ce sujet (Carlisle, 2010; Bowers et al., 2010). Conformément aux recommandations de certains 
chercheurs (Allal, 1997; Anglin, 1993), la coopération et l'approche de résolution de problème ont été privilégiées dans la réalisation des activités du programme. L'enchainement des activités est facilité par la trame de l'histoire d'une famille qui ajoute une valeur ludique et motivationnelle au programme.

\section{Méthodologie}

\section{Participants}

Les participants de cette recherche sont les élèves arabophones de $3^{\mathrm{e}}$ et de $4^{\mathrm{e}}$ année de deux écoles de Montréal caractérisées par une forte concentration de cette population et ayant des indices de milieu socio-économique comparables (27,47 et 22,95; (MELS, 2006). Ces indices mettent en évidence que les écoles participantes ont un niveau de défavorisation élevé.. Les données fournies par les parents attestent que les participants ont commencé à parler le français avant le début de la scolarisation (la moyenne du temps d'exposition au français est de 5 ans et 8 mois, pour les élèves de $3^{\mathrm{e}}$ année, et de 6 ans et 2 mois, pour ceux de $4^{\mathrm{e}}$ année). La langue parlée à la maison de tous les participants est l'arabe. Pour ce qui est de leur connaissance de l'arabe écrit, aucune mesure n'a été administrée. Or, nous savons que 28 d'entre eux suivent le programme d'enseignement dans les langues d'origine (PÉLO) en arabe à une fréquence de quatre jours par semaine pendant des sessions de 30 minutes depuis leur première année.

Selon un devis quasi-expérimental, les participants de chaque école ont été assignés à un groupe de recherche. Les élèves de l'école qui a eu les plus faibles résultats en conscience morphologique et en identification des mots au prétest ont été assignés au groupe expérimental. Le tableau 1 décrit notre échantillon. 
Tableau 1 Description de l'échantillon

\begin{tabular}{l|l|l|l|l|l}
\hline Groupe et niveau & $\begin{array}{l}\text { Nombre de } \\
\text { sujets }\end{array}$ & Filles & Garçons & $\begin{array}{l}\text { Âge } \\
\text { moyen }\end{array}$ & Écart-type \\
\hline Expérimental $3^{\mathrm{e}}$ année & 21 & 10 & 11 & 8,88 & 0,37 \\
\hline Expérimental $4^{\mathrm{e}}$ année & 10 & 6 & 4 & 9,89 & 0,54 \\
\hline Contrôle $3^{\mathrm{e}}$ année & 11 & 6 & 5 & 8,93 & 0,39 \\
\hline Contrôle $4^{\mathrm{e}}$ année & 10 & 3 & 7 & 9,9 & 0,52 \\
\hline
\end{tabular}

\section{Mesures}

Conscience morphologique. Afin d'«aligner les mesures expérimentales à l'instruction» (Carlisle, 2010), nous avons administré un test expérimental ciblant, tout comme le programme d'intervention, la morphologie dérivationnelle. Il s'agit d'un test collectif composé de 3 tâches contenant chacune 10 phrases à compléter. Quoiqu'utilisé largement dans les recherches (Carlisle, 1995; Fowler \& Liberman, 1995), ce test comporte un biais puisque le participant peut recourir au contexte sémantique pour compléter la phrase. Afin de limiter ce biais, le mot de la même famille que le mot cible a été présenté en gras pour l'ensemble des tâches (Un poissonnier vend du (poisson). Dans les deux premières tâches, on demande aux élèves de compléter les phrases en produisant un mot dérivé et en extrayant la base. Les items de chacune des deux premières tâches ont été choisis selon qu'ils correspondaient à la condition phonologique neutre (marier/mariage) ou à la condition phonologique complexe (faire/infaisable). L'ordre de présentation des items dans les deux conditions a été randomisé avant d'être présenté aux participants. La troisième tâche porte sur la dérivation des pseudo-mots (morer/démorer). L'ensemble des tâches fait appel aux préfixes et aux suffixes ciblés par l'intervention. Chaque bonne réponse compte pour un point. Les indices de fidélité sont $\alpha=.975$ pour la dérivation des mots, $\alpha=.970$ pour l'extraction de la base et $\alpha=.912$ pour la dérivation des non-mots. Le test a préalablement été validé dans une classe de $3^{\mathrm{e}}$ année d'une école du même quartier que les écoles participant à la recherche. 
Identification des mots. Le test MIM (mesure d'identification des mots) de la batterie BELEC (Mousty, Leybaert, Alegria, Content, \& Morais, 1994) retenu pour cette variable permet de mesurer de manière appropriée les effets de l'intervention sur la capacité à identifier les mots écrits. Ce test est composé de soixante-douze mots répartis également en mots courts (monomorphémiques, $n=36$ ) et en mots longs (plurimorphémiques, $n=36$ ), dont la fréquence et les particularités orthographiques sont rigoureusement contrôlées. Les mots de ces séries ne sont pas utilisés au cours de l'intervention; par contre, la majorité des affixes des mots plurimorphémiques ont été vus lors des activités du programme. Le test est composé de deux séries que les auteurs considèrent équivalentes. La série A de MIM est utilisée au prétest, tandis que la série B est utilisée au post-test. Le temps de réponse et la précision sont mesurés conformément au protocole de passation de l'épreuve.

Comme l'identification des mots est influencée par la conscience phonologique, les capacités cognitives et la dénomination rapide (Bowey, 2005), ces variables ont également été mesurées afin de bien isoler les effets du programme.

Conscience phonologique. L'épreuve de conscience phonologique, extraite de BÉLÉC (Mousty et al. 1994), exige que l'élève fasse successivement deux manipulations: l'isolement du premier phonème de deux mots et la fusion de ces phonèmes pour former une syllabe. Il est composé de 16 paires de mots qui contiennent chacune une discordance entre le phonème initial du mot et le phonème correspondant à la première lettre du mot (ex., dans autobus, le phonème /o/ du mot entendu est différent du phonème /a/ correspondant à la première lettre de ce mot à l'écrit). Chaque réponse qui correspond à l'acronyme formé des initiales à l'oral compte pour un point. La réponse qui tient compte de l'orthographe des mots ne compte pas comme une bonne réponse (ex. / ka/ pour cher Auguste est considéré erroné).

Intelligence non verbale. Pour mesurer les capacités cognitives, nous avons administré, comme d'autres études (Kirby et al., 2012) les trois premières matrices de Raven (1983). Chaque matrice comporte 12 exercices présentés sous forme d'images. Le participant doit 
choisir une image parmi les six images proposées pour compléter un modèle donné. Il y a seulement une bonne réponse à laquelle on accorde un point.

Dénomination rapide. Le test Rapid Automatic Naming (RAN) de Wolf et Denckla (2005) est composé de quatre sous-tests de dénomination de lettres (RANL-1 et RANL-2) et de chiffres (RAND-1 et RAND-2). L'élève est invité à nommer le plus rapidement possible les lettres et les chiffres. Le temps mis à réaliser chaque sous-test de même que la précision de chaque réponse sont mesurés pour ce test.

\section{Variable indépendante}

Au début, le programme de conscience morphologique a été validé auprès d'experts, d'enseignants et de conseillers pédagogiques. Après avoir apporté les modifications suggérées, la chercheuse a, par la suite, validé le programme dans une classe de $3^{\mathrm{e}}$ année d'une école dont les caractéristiques socio-économiques sont similaires à celles des deux écoles qui participent à la recherche. Après cette validation, des modifications mineures ont été apportées au programme. Les activités ont été réalisées une fois par semaine, pendant dix semaines. Chaque session a duré 50 à 60 minutes. Elles ont été offertes par la chercheuse à cinq groupes-classes de 18 à 20 élèves assignés au groupe expérimental. Le groupe contrôle a suivi un enseignement régulier qui consistait à réaliser les activités prévues dans le manuel de français. Ce manuel était le même que celui utilisé dans les classes du groupe expérimental. De plus, sur la base des informations fournies par les enseignantes des classes de la condition témoin, aucune activité visant la morphologie dérivationnelle n'a été proposée aux élèves entre le prétest et le post test.

\section{Procédure}

Tout d'abord, des mesures des variables de contrôle et dépendantes ont été prises auprès des participants des deux groupes de recherche au mois de février, avec une différence d'une semaine entre les deux groupes. Cette différence a été respectée lors de la passation des post-tests au mois de mai. L'intervention en conscience morphologique a été réalisée durant la période mi-février-début mai. Finalement, des mesures ont été prises après l'intervention auprès des participants des deux groupes. Le test de conscience 
morphologique a été administré en modalité collective dans chacun des locaux des dix classes participantes. Les autres tests ont été administrés, en modalité individuelle, dans un local tranquille de l'école. Pour le test de conscience morphologique, trois exemples pour chacune des tâches ont été fournis préalablement au tableau. Par la suite, tel que suggéré par Berninger et al. (2010), les élèves ont complété les phrases que la chercheuse lisait afin de contrer le biais relié aux difficultés de lecture des certains élèves. Le test de Raven (1983) a été administré en sous-groupes de 7 à 9 élèves dans un local tranquille, tel qu'il est recommandé pour ce groupe d'âge. Les tests de dénomination rapide (RAN, Wolf et Denckla, 2005), d'identification des mots (BELEC, 1994) et de conscience phonologique (BELEC, 1994) ont été effectués individuellement et conformément au protocole de passation.

\section{Résultats}

Nous présentons tout d'abord les résultats des deux groupes de recherche au prétest (tableau 2) qui permettent de constater que le groupe contrôle performe généralement mieux que le groupe expérimental. Or, la différence n'est pas significative pour l'identification des mots et la conscience morphologique.

Tableau 2 Résultats des deux groupes au prétest

\begin{tabular}{l|c|c|c|c|c|c}
\hline & \multicolumn{2}{|c|}{$\begin{array}{c}\text { Groupe expérimental } \\
\mathrm{n}=31\end{array}$} & \multicolumn{2}{c|}{$\begin{array}{c}\text { Groupe contrôle } \\
\mathrm{n}=21\end{array}$} & & \\
& $\mathrm{M}$ & $\mathrm{ÉT}$ & $\mathrm{M}$ & ÉT & $\mathrm{t}$ & $\mathrm{p}$ \\
\hline Conscience phonologique & 11.71 & 2.85 & 13.19 & 1.60 & 2.155 & .036 \\
\hline Intelligence non verbale & 21.52 & 7.87 & 27.95 & 5.47 & 3.249 & 0,002 \\
\hline Dénomination rapide & 29.85 & 5.11 & 27.08 & 4.58 & 1.999 & .051 \\
\hline Conscience morphologique & 12,26 & 5.651 & 15.05 & 5.210 & 1.802 & .078 \\
\hline IMÉ courts (précision) & $32.42(90.1 \%)$ & 2.849 & $32.48(90.2 \%)$ & 2.502 & .074 & .941 \\
\hline IMÉ longs (précision) & $27.97(77,7 \%)$ & 5.58 & $30.00(83.3 \%)$ & 2.76 & 1.544 & .129 \\
\hline IMÉ courts (rapidité) & 32.55 & 6.51 & 34.06 & 11.71 & .594 & .555 \\
\hline IMÉ longs (rapidité) & 72.00 & 21.17 & 66.38 & 16.82 & 1.017 & .314 \\
\hline
\end{tabular}


Les résultats obtenus au post-test aux mesures des variables dépendantes sont présentés au tableau 3. Nous les avons comparés avec les résultats au prétest et ensuite procédé à des analyses de variance et de covariance à mesures répétées intersujets (tableau 4), des analyses très puissantes pour mesurer les effets d'une intervention dans le cas des recherches à petit échantillon (Larson-Hall, 2010). La taille de l'effet y est présentée également.

Tableau 3 Résultats des deux groupes au post test

\begin{tabular}{l|c|c|c|c}
\hline & \multicolumn{2}{|c|}{$\begin{array}{c}\text { Groupe expérimental } \\
\mathrm{n}=31\end{array}$} & \multicolumn{2}{c}{$\begin{array}{c}\text { Groupe contrôle } \\
\mathrm{N}=21\end{array}$} \\
\hline & $\mathrm{M}$ & ÉT & $\mathrm{M}$ & ÉT \\
\hline Épreuves morphologiques & $19.87(66.23 \%)$ & 5.691 & $15.10(50.33 \%)$ & 4.437 \\
\hline IMÉ courts (précision) & $34.52(95.88 \%)$ & 1.59 & $32.81(91.13)$ & 1.91 \\
\hline IMÉ longs (précision) & $32.48(90.22 \%)$ & 3.38 & $30.38(84.38 \%)$ & 3.35 \\
\hline IMÉ courts (rapidité) & 30.49 & 7.51 & 31.22 & 7.35 \\
\hline IMÉ longs (rapidité) & 55.45 & 16.15 & 55.87 & 10.74 \\
\hline
\end{tabular}

Comparé au tableau 2, ce tableau met en évidence des résultats supérieurs au post test des deux groupes de recherche sur les variables mesurées. Cependant, après l'analyse de variance à mesures répétées, dont les résultats sont présentés dans le tableau 4, il ressort qu'il y a un effet d'interaction tempsxgroupe ce qui signifie que les élèves du groupe expérimental performent significativement mieux que le groupe contrôle aux tâches de conscience morphologique $(F(1,51)=37.779$ à $p<.000)$. La taille de l'effet est de $\eta^{2}=.43$. $\mathrm{Si}$, en référence à Howell (2008), cet effet du programme de conscience morphologique peut être considéré de grande taille, il se situe dans la moyenne des effets de telles interventions qui s'élève à .44 (Goodwin \& Ahn, 2013). 
Tableau 4. Résultats des ANOVA et des ANCOVA après le contrôle de la conscience phonologique, la dénomination rapide et l'intelligence non verbale

\begin{tabular}{l|c|c|c|c|c|c}
\hline & \multicolumn{2}{|c|}{$\begin{array}{c}\text { ANOVA à } \\
\text { mesures répétées }\end{array}$} & $\begin{array}{c}\text { Taille } \\
\text { de } \\
\text { l'effet }\end{array}$ & \multicolumn{2}{c|}{ ANCOVA } & $\begin{array}{c}\text { Taille } \\
\text { de } \\
\text { l'effet }\end{array}$ \\
\hline & $F$ & $p$ & $\eta^{2}$ & $F$ & $p$ & $\eta^{2}$ \\
\hline Épreuves morphologiques & 37.779 & .000 & .43 & & & \\
\hline IMÉ courts (précision) & 8.637 & .005 & .147 & 4.109 & 0.48 & .080 \\
\hline IMÉ longs (précision) & 25.687 & .000 & .339 & 14.637 & .000 & .237 \\
\hline IMÉ courts (rapidité) & 0.85 & .772 & .002 & .692 & .410 & .015 \\
\hline IMÉ longs (rapidité) & 3.648 & .062 & .068 & .615 & .437 & 0.13 \\
\hline
\end{tabular}

Pour ce qui est de l'identification des mots, les deux groupes ont amélioré leurs scores aux quatre aspects vérifiés, c'est-à-dire la précision et la rapidité des mots courts et des mots plurimorphémiques. L'analyse de variance révèle que le groupe expérimental performe significativement mieux que le groupe contrôle au regard de la précision de l'identification des mots plurimorphémiques $(F(1,51)=25.687$ à $\mathrm{p}=.000)$ mais il ne les lit pas significativement plus rapidement que le groupe contrôle $(F(1,51)=3.648$ à $p=.062)$. Le tableau 4 permet de remarquer que l'effet significativement positif de l'intervention sur la précision des mots plurimorphémiques est maintenu même après avoir effectué des ANCOVA en entrant les variables de contrôle, c'est-à-dire la conscience phonologique, la dénomination rapide et l'intelligence non verbale $(F(1,51)=14.637$ à $p=.000)$.

\section{Discussion}

Notre objectif était d'évaluer les effets de l'intervention en conscience morphologique sur l'identification des mots écrits plurimorphémiques. Notre hypothèse postulait que les élèves du groupe expérimental ayant bénéficié du programme de conscience morphologique auraient une performance significativement supérieure à celle du groupe contrôle en identification des mots plurimorphémiques, en termes de précision et de rapidité. Afin de vérifier cette hypothèse, nous avons procédé à des analyses de variance et de covariance à mesures répétées intersujets. Ces analyses indiquent que les élèves du groupe expérimental présentent une performance significativement supérieure à celle du 
groupe contrôle, lors du post-test, en ce qui concerne la précision de l'identification de mots plurimorphémiques. Ces résultats corroborent ceux d'autres recherches qui montrent des effets significativement positifs de l'intervention en conscience morphologique sur l'identification des mots dans d'autres langues en L1 (Lyster, 2002; Nunes et al. 2003). L'amélioration significative de la précision de l'identification des mots plurimorphémiques chez les élèves du groupe expérimental peut être expliquée à la lumière de modèles fonctionnels et développementaux de l'identification des mots. Ainsi, conformément au modèle des deux voies d'accès (Chialant \& Caramazza, 1995), les participants du groupe expérimental qui avaient une plus grande expérience que le groupe contrôle avec les affixes contenus dans les mots du test, la majorité de ces affixes étant travaillés lors du programme, ont mieux identifié les mots. Selon le modèle de la qualité lexicale de Reichle et Perfetti (2003), cette expérience conduit à des représentations orthographiques plus précises. En effet, lors des activités, les élèves étaient fréquemment invités à réfléchir et à discuter à propos de la forme orthographique des mots. Cette attention particulière à la forme des mots écrits contribuerait à la construction de structures orthographiques qui permettent l'identification précise des mots écrits (Ehri, 2005; Seymour 2005). Dans le cas de cette recherche, la sensibilité à la structure morphologique des mots et l'éventuelle construction des structures orthographiques des affixes vus durant le programme pourraient expliquer la plus grande précision de l'identification des mots plurimorphémiques chez les élèves du groupe expérimental. Cet effet serait, théoriquement parlant, plus tangible chez une population qui aurait des représentations orthographiques peu précises étant donné que leur expérience avec l'écrit est divisé entre deux langues, le français et l'arabe, et que l'expérience avec l'arabe les amènerait à s'appuyer davantage sur les consonnes (Randall and Meara, 1988). Toutefois, comme la performance des participants en identification des mots, en arabe, n'a pas été mesurée, il convient d'être prudent quant à cette interprétation, même si nous savons que la majorité de ceux-ci (28 sur 52) suivent depuis au moins deux ans le cours d'arabe. De plus, le manque d'un groupe de participants dont le français est la langue première invite à la prudence. 
Concernant la performance en termes de rapidité d'identification des mots plurimorphémiques, la différence entre les deux groupes n'est pas significative, même si le groupe expérimental a davantage amélioré sa performance par rapport au groupe contrôle. Cette absence d'effet significatif du programme sur la rapidité d'identification peut s'expliquer de deux façons. Premièrement, la rapidité étant associée à l'automatisation des processus (Ehri, 2005), la courte durée de cette intervention n'aurait pas permis aux élèves d'atteindre un niveau d'automatisation suffisant de ces processus. D'ailleurs, Nagy et al. (2006) ont mis en évidence que la conscience morphologique influence tardivement la rapidité de l'identification des mots écrits. Toutefois, étant donné qu'aucune intervention en conscience morphologique n'a été documentée dans le cadre de leur recherche, il convient également d'envisager une autre explication liée à l'entrainement de la capacité à lire rapidement les mots plurimorphémiques dans le cadre de notre programme. En fait, une seule activité d'une dizaine de minutes prévue à cet effet ne pourrait pas améliorer significativement la rapidité à identifier les mots plurimorphémiques chez les élèves du groupe expérimental. Il est donc possible que le nombre d'activités visant l'identification rapide de mots écrits plurimorphémiques ait été insuffisant pour avoir un effet sur la rapidité de traitement de ces items

Nos résultats en L2 supportent les recommandations (Gombert et al., 2000; Nunes, Bryant, \& Barros, 2012) à l'effet que des activités sur la formation des mots aideraient les lecteurs intermédiaires. En revanche, la rapidité d'identification de ces mots n'a pas été améliorée de manière significative par le programme. Considérant le fait que la rapidité de l'identification des mots chez les élèves du groupe expérimental s'est améliorée plus que chez ceux du groupe contrôle, il serait judicieux de vérifier si le fait d'accorder plus de temps à l'entrainement de la rapidité aurait un effet significatif.

\section{Conclusion}

Cette recherche auprès d'une population allophone scolarisée en français apporte des résultats encourageants qui s'ajoutent à ceux d'autres recherches causales ayant démontré la contribution de la conscience morphologique dans l'identification des mots en L1. Nos résultats s'ajoutent au corpus de recherches qui mettent en évidence le rôle crucial de la conscience morphologique dans l'identification des mots écrits et appuient la 
recommandation d'inclure l'entrainement de la conscience morphologique dans l'enseignement de la lecture (Berninger et al., 2010). Par ailleurs, ces résultats corroborent les résultats d'autres recherches soulignant que les élèves non natifs de la langue d'enseignement développent des capacités d'identification des mots écrits qui suivent une trajectoire semblable à celle des natifs (Lesaux, Geva, Koda, Siegel, \& Shanahan, 2008).

Malgré les résultats positifs obtenus, il importe de mentionner certaines limites de cette recherche. Premièrement, le nombre restreint de sujets limite la généralisation des résultats. Deuxièmement, le biais relié à l'effet Hawthorne n'a pas été contrôlé, puisque le groupe contrôle n'a pas reçu d'intervention systématique portant sur une autre habileté. Troisièmement, n'ayant pas mesuré l'identification des mots en arabe, nous n'avons pu avancer davantage dans nos interprétations.

Finalement, la présente recherche informe le milieu de l'éducation quant trois pôles du triangle didactique. En effet, elle souligne les besoins de soutien de la population arabophone scolarisée en français (pôle élève) en ce qui concerne l'identification des mots plurimorphémiques (pôle objet). Compte tenu des résultats significativement positifs de cette étude, il serait approprié de proposer des activités de conscience morphologique à ladite population et ce, dès la $3^{\mathrm{e}}$ année du primaire, moment où les élèves sont exposés à une augmentation substantielle de mots plurimorphémiques dans les textes qu'ils lisent (Anglin, 1993). De plus, la présente étude renseigne sur les modalités d'une intervention didactique en conscience morphologique telles que le contenu, la fréquence, la durée et le niveau scolaire (pôle enseignant). De futures recherches de plus longue durée seraient toutefois nécessaires pour vérifier les effets d'une telle intervention sur la rapidité de l'identification des mots plurimorphémiques.

\section{Références}

Allal, L. (1997). Acquisition de l'orthographe en situation de classe. In L. Rieben, M. Fayol \& C. Perfetti (Eds.), Des orthographes et leur aquisition (pp. 181-206). Lausanne/Paris: Delachaux et Niestlé.

Anglin, J. (1993). Vocabulary development : A morphological analysis. Monographs of the Society of Research in Child Development (Vol. 58, pp. 1-166). Chicago: Society for Research in Child Development. 
Besse, A.-S., Demont, E., \& Gombert, J.-É. (2007). Effet des connaissances linguistiques en langue maternelle (arabe vs portugais) sur les performances phonologiques et morphologiques en français langue seconde. Psychologie française, 52(1), 89105.

Bowers, P. (Ed.). (2009). Teaching how the written word works. Kingston: Wordworks literacy centre.

Bowey, J. A. (2005). Predicting individual differences in learning to read. In M. J. Snowling \& C. Hulme (Eds.), The science of reading : A handbook (pp. 155-172). Lon: Blackwell Publishing.

Braunger, J., \& Lewis, J. P. (Eds.). (1998). Building a knowledge base in reading, . Newark: Northwest regional educational laboratory's NCTE, IRA.

Carlisle, J. (1995). Morphological awareness and early reading achievement. In L. B. Feldman (Ed.), Morphological aspects of language processing (pp. 189-209). Hillsdale: Erlbaum.

Carlisle, J. (2000). Awareness of the Structure and Meaning of Morphologically Complex Words: Impact on Reading. Reading and Writing: An interdisciplinary journal, 12(3-4), 169-190.

Carlisle, J. (2010). Review of Nunes, T., and Bryant, P. (2006). Improving literacy by teaching morphemes. London: Routledge. Reading and Writing, 23(2), 257-262. doi: 10.1007/s11145-008-9151-9.

Casalis, S., Dusautoir, M., Cole, P., \& Ducrot, S. (2009). Morphological Effects in Children Word Reading: A Priming Study in Fourth Graders. British Journal of Developmental Psychology, 27(3), 761-766.

Casalis, S., \& Louis-Alexandre, M.-F. (2000). Morphological Analysis, Phonological Analysis and Learning To Read French: A Longitudinal Study. Reading and Writing: An interdisciplinary journal, 12(3-4), 303-335.

Catach, N. (1978). L'orthographe. Que sais-je? Paris: Presses universitaires de France.

Chialant, D., \& Caramazza, A. (1995). Where is morphology and how is it processed? The case of written word recognition. In L. B. Feldman (Ed.), Morphological aspects of language processing (pp. 55-76). Hillsdale, NJ, England: Lawrence Erlbaum Associates.

Colé, P., Royer, C., Leuwers, C., \& Casalis, S. (2004). Les connaissances morphologiques dérivationnelles et l'apprentissage de la lecture chez l'apprentilecteur français du CP au CE2. Année psychologique, 104, 701-750.

Deacon, S., \& Kirby, J. (2004). Morphological awareness: Just "more phonological"? The roles of morphological and phonological awareness in reading development. Applied Psycholinguistics, 25(2), 223.

Deacon, S., Parrila, R., \& Kirby, J. (2006). Processing of Derived Forms in HighFunctioning Dyslexics. Annals of Dyslexia, 56(1), 103-128.

Deacon, S., Whalen, R., \& Kirby, J. (2011). Do Children See the "Danger" in "Dangerous"? Grade 4, 6, and 8 Children's Reading of Morphologically Complex Words. Applied Psycholinguistics, 32(3), 467-481.

Ehri, L. (2005). Development of sight word reading: Phases and findings. In M. Snowling \& C. Hulme (Eds.), The Science of Reading: A Handbook (pp. 135-154). Malden, Oxford, Carlton: Blackwell Publishing. 
Elbro, C., \& Arnbak, E. (1996). The role of morpheme recognition and morphological awareness in dyslexia. Annals of Dyslexia, 46, 209-240.

Fowler, C., \& Liberman, I. (1995). The role of phonology and orthography in morphological awareness. In L. Feldman (Ed.), Morphological aspects of language processing (pp. 157-188). Hillsdale: Erlbaum.

Geva, E. (2009). Apprendre à lire dans une langue seconde: recherche, implications et recommandations pour les services (Publication no. http://www.enfantencyclopedie.com/documents/GevaFRxp.pdf). from Encyclopédie sur le développement des jeunes enfants

Gombert, J.-É., Colé, P., Valdois, S., Goigoux, R., Mousty, P., \& Fayol, M. (Eds.). (2000). Enseigner la lecture au cycle 2. Paris: Nathan.

Goodwin, A. P., \& Ahn, S. (2013). A Meta-Analysis of Morphological Interventions in English: Effects en Literacy Outcomes for School-Age Children. Scientific Studies of Reading, 17(4), 257-285.

Howell, D. (Ed.). (2008). Méthodes statistiques en sciences humaines. Bruxelles: De boeck.

Jaffré, J.-P. (1999). Orthographe et principes d'écriture en français. Rééducation orthophonique, 200(décembre), 25-34.

Jaffré, J.-P., \& Fayol, M. (1997). Orthographes des systèmes aux usages. Paris: Dominos Flammarion.

Jaffré, J.-P., \& Fayol, M. (2006). Orthography and literacy in french. In R. M. Joshi \& P. G. Aaron (Eds.), Handbook of orthography and literacy (pp. 81-104). Mahwah, New Jersey, London: Lawrence Erlbaum Associates.

Kirby, J., Deacon, S., Bowers, P., Izenberg, L., Wade-Woolley, L., \& Parrila, R. (2011). Children's morphological awareness and reading ability. Reading and Writing, 122. doi: 10.1007/s11145-010-9276-5

Koda, K. (2004). Insights into Second-Language Reading : A cross-linguistic approach. Cambridge: University Press.

Larson-Hall, J. (Ed.). (2010). A guide to doing statistics in second language research using SPSS. New York and London: Routledge Taylor and Francis group.

Lesaux, N., Geva, E., Koda, K., Siegel, L., \& Shanahan, T. (2008). Development of literacy in second-language learners. In D. August \& T. Shanahan (Eds.), Developping Reading and writing in second-language learners: Lessons from the Report of the National Literacy Panel on Language-Minority Children and Youth. (pp. 27-60). New York, Abingdon: Routledge, Center for Applied Linguistics, International Reading Association.

Lyster, S. A.-H. (2002). The effects of morphological versus phonological awareness training in kindergarten on reading development. Reading and Writing: An interdisciplinary journal, 15(3-4), 261-294.

Mahony, D., Singson, M., \& Mann, V. (2000). Reading ability and sensitivity to morphological relations. Reading and Writing: An interdisciplinary journal, 12(34), 191-218.

McCarthy, J., \& Prince, A. (1990). Foot and Word in Prosodic Morphology: the Arabic Broken Plural. Natural Language and Linguistic Theory, 8, 209-283. 
MELS. (2006). Portrait scolaire des élèves issus de l'immigration : de 1994-1995 à 20032004. In G. d. Québec (Ed.). Québec.

Mousty, P., Leybaert, J., Alegria, J., Content, A., \& Morais, J. (1994). BELEC : Batterie d'évaluation du langage écrit et de ses troubles. . In J. Grégoire \& B. Pierart (Eds.), Evaluer les troubles de la lecture. Les nouveaux modèles théoriques et leurs implications diagnostiques (pp. 127-145). Bruxelles: De Boeck.

Nagy, W., Berninger, V., \& Abbot, R. (2006). Contributions of Morphology beyond Phonology to Literacy Outcomes of Upper Elementary and Middle-School Students. Journal of Educational Psychology, 98(1), 134-147.

Nunes, T., Bryant, P., \& Barros, R. (2012). The Development of Word Recognition and Its Significance for Comprehension and Fluency. Journal of Educational Psychology, 104(4), 959-973.

Nunes, T., Bryant, P., \& Olsson, J. (2003). Learning phonological and morphological spelling rules: An intervention study. Scientific Studies of Reading, 7(3), 289-307.

Raven, J. C. (Ed.). (1983). Standard Progressive Matrices. London: H.K. Lewis.

Reichle, E., \& Perfetti, C. (2003). Morphology in Word Identification: A WordExperience Model that Accounts for Morpheme Frequency Effects. Scientific Studies of Reading, 7(3), 219-237.

Schwiebert, C., Green, L., \& McCutchen, D. (2002). The Contribution of Morphology to Reading and Spelling Achievement (pp. 29).

Sénéchal, M., \& Kearnan, K. (2007). The role of morphology in reading and spelling. Advances in child development and behavior, 35, 297-326.

Seymour, P. H. K. (2005). Early Reading Development un European Orthographies. In M. J. Snowling \& C. Hulme (Eds.), The science of reading : A handbook (pp. 296-315). Malden, Oxford, Carlton: Blackwell Publishing.

Singson, M., Mahony, D., \& Mann, V. (2000). The relation betwenn reading ability and morphological skills: evidence from derivational suffixes. Reading and Writing: An interdisciplinary journal, 12(3-4), 219-252.

Verhoeven, L., \& Perfetti, C. (2003). Introduction du this special issue: the role of morphology in learning to read. Scientific Studies of Reading, 7(3), 209-217.

Verhoeven, L., \& Perfetti, C. (2011). Morphological processing in reading acquisition: A cross-linguistic perspective. Applied Psycholinguistics, 32(3), 457-466.

Verhoeven, L., \& Schreuder, R. (2011). Morpheme frequency effects in Dutch complex word reading: A developmental perspective. Applied Linguistics, 32, 483-498.

Wolf, M., \& Denckla, M. B. (2005). Rapid automatized naming abd rapid alternating stimulus tests. Examiner's manual. Texas: Proed.

Ziegler, J. C., \& Goswami, U. (2005). Reading acquisition, developmental dyslexia and skilled reading across languages: A psycholinguistic grain size theory. Psycological Bulletin, 131(1), 3-29. 


\section{Références des auteurs:}

Anila Fejzo a travaillé en tant qu'enseignante auprès de différentes populations d'élèves, au primaire, au secondaire, en langue première et en langue seconde. Ses intérêts de recherche portent sur le développement de la lecture et de l'écriture chez les élèves du primaire. Plus spécifiquement, elle s'intéresse aux relations entre le développement métalinguistique et le développement de la littératie. Dans cette optique, elle investigue les effets d'interventions didactiques ciblant des habiletés métalinguistiques sur les capacités à lire et à écrire.

Lucie Godard a d'abord été orthophoniste en milieu scolaire pendant 14 ans. Elle s'intéresse aux difficultés d'apprentissage de la langue orale et écrite et aux premiers apprentissages en lecture et en écriture. Ses recherches actuelles portent sur l'implantation du modèle à trois niveaux de réponse à l'intervention pour les premiers apprentissages en écriture et en lecture auprès d'élèves de milieux défavorisés et d'élèves allophones. Elle a participé à plusieurs recherches menées auprès d'élèves allophones scolarisés en français. Ses travaux ont contribué à décrire les caractéristiques de ces apprenants et à dégager des pistes didactiques adaptées à leurs besoins.

Line Laplante est spécialiste de l'évaluation et de l'intervention relatives aux difficultés spécifiques d'apprentissage de la lecture-écriture. Ses travaux de recherche s'inscrivent dans la logique du modèle de réponse à l'intervention avec protocole standard (RàI/RTI); ils visent à évaluer l'impact de l'intervention universelle (niveau 1), de l'intervention supplémentaire ciblée (niveau 2) et de l'intervention sur mesure (niveau 3) sur la prévention des difficultés au regard des premiers apprentissages en lecture et en écriture.

Les trois auteures sont professeures au département de didactique des langues de l'Université du Québec à Montréal

Email: fejzo.anila@uqam.ca (auteur principal)

Pour citer cet article:

Fejzo, A., Godard, L., \& Laplante, L. (2014). Les effets d'un programme de conscience morphologique sur l'identification des mots en français. Bellaterra Journal of Teaching \& Learning Language \& Literature, $7(4), 15-37$. 